\title{
Sensitivity Patterns of Isolated Salmonella Typhi in Children with Typhoid Fever
}

\author{
Sk. Mohammad Ali Rashed ${ }^{1, ~ *, ~ M u r s h i d a ~ A k h t e r ², ~ M d . ~ R u h u l ~ A m i n ~}{ }^{3}$, Mahbub Mutanabbi ${ }^{4}$, \\ Choudhury Ali Kawser ${ }^{4}$ \\ ${ }^{1}$ Department of Pediatrics, Upazila Health Complex, Charghat, Rajshahi, Bangladesh \\ ${ }^{2}$ Sir Salimullah Medical College \& Mitford Hospital, Dhaka, Bangladesh \\ ${ }^{3}$ Department of Pediatrics, Rajshahi Medical College Hospital, Rajshahi, Bangladesh \\ ${ }^{4}$ Department of Pediatrics, Bangabandhu Sheikh Mujib Medical University (BSMMU), Dhaka, Bangladesh
}

Email address:

sk.md.alirashed@gmail.com (Sk. M. A. Rashed)

${ }^{*}$ Corresponding author

\section{To cite this article:}

Sk. Mohammad Ali Rashed, Murshida Akhter, Md. Ruhul Amin, Mahbub Mutanabbi, Choudhury Ali Kawser. Sensitivity Patterns of Isolated Salmonella Typhi in Children with Typhoid Fever. American Journal of Pediatrics. Vol. 7, No. 2, 2021, pp. 44-48.

doi: 10.11648/j.ajp.20210702.11

Received: February 13, 2021; Accepted: February 26, 2021; Published: April 1, 2021

\begin{abstract}
Background and objective: Enteric fever is a common febrile illness and one of te major health problem in Bangladesh. So the present study was conducted to determine the demographic characteristics, clinical profile, hematological feature and current sensitivity patterns of isolated $\mathrm{S}$ typhi in patients suffering from enteric fever in some of the tertiary care hospitals in Dhaka. Materials \& Methods: This was a cross sectional study conducted in the Department of Pediatrics, Bangabandhu Sheikh Mujib Medical University (BSMMU), Dhaka Medical College Hospital (DMCH) and SSMCH - Sir Salimullah Medical College \& Hospital (SSMCH), Dhaka, Bangladesh for a period of one year from July 2015 to June 2016. A total number of 100 children with enteric fever were studied and diagnose were based on clinical features, Wedel test and blood culture. Results: Out of 100 diagnosed enteric fever children, $60(60 \%)$ were male and $40(40 \%)$ were female. Fever was present in all patients. Other most common manifestation are loss of appetite, vomiting, diarrhea, headache and constipation. Coated tongue was the predominant examination finding followed by hepatomegaly, splenomegaly and abdominal tenderness. Sensitivity to ceftriaxone was $100 \%$ in our study while resistance to nalidixic acid was $90 \%$. MDR S.typhi is still a threat but there was re-emergence of sensitivity to Cotrimoxazole and Chloramphenicol. Conclusion: The study concluded that clinical pattern of typhoid fever conforms well to that of known pattern of the disease in children. Blood culture profile shows widespread emergence of nalidixic acid related resistant strains and the sporadic occurrence of cephalosporin resistant strains of S. Typhi. However, the first line antibiotics such as chloramphenicol and cotrimoxazole still have a role to play in the treatment of typhoid due to re-emergence of sensitivity.
\end{abstract}

Keywords: Typhoid Fever, Antimicrobial, Sensitivity, Isolated, Salmonella Typhi, Pediatrics, Cotrimoxazole

\section{Introduction}

Enteric fever, an acute generalized infection may affect nearly all the systems but more commonly the reticuloendothelial system, intestinal lymphoid tissue, and the gallbladder. It includes 'typhoid fever' caused by Salmonella enterica serovar Typhi (Salmonella typhi) and 'paratyphoid fever' caused by Salmonella paratyphi A or Salmonella paratyphi B (or rarely, Salmonella paratyphi C). [1] Humans are the natural host and reservoir of these organisms. Salmonella can survive for days in groundwater or seawater and for months in contaminated eggs and frozen oysters. [1] The infectious dose varies between $10^{3}-10^{6}$ organisms given orally. [2] Transmission of infection occurs by ingestion of food or water contaminated with faeces. Other established risk factors include recent contact with a typhoid patient or carrier, eating ice cream, flavored iced drinks or food from street vendors, and raw fruit and vegetables grown in fields fertilized 
with sewage. [2] Because of the provision of clean water and good sewage system, it is a sporadic disease in developed countries and occurs mainly in travelers returning from endemic regions. Today, most of the burden of the disease is limited to the developing countries where standards of hygiene and sanitation remain poor. [3]. Despite the use of antibiotics and the development of newer antimicrobial agents, enteric fever continues to be a major public health problem causing high morbidity and mortality in developing countries. [4] The first-line drugs ampicillin, chloramphenicol, and cotrimoxazole were used as a standard treatment regimen for enteric fever until the mid-1980s. [5] However, the indiscriminate use of these drugs and acquisition of plasmid-mediated resistance led to the development of typhoid resistant to ampicillin, chloramphenicol, and cotrimoxazole -multi-drug resistant (MDR) typhoid - by end of $80^{\prime 1}$. The knowledge of the prevalence of Salmonella serovars and determination of their antimicrobial resistance pattern are very important for the guidance of clinical management of enteric fever. [6] This overall assessment is conducted over the profile of patients, and it is done to bring in the results how the Typhoid Fever and Antimicrobial Sensitivity patterns of Isolated Salmonella Typhi are related. Thus, this may help in developing appropriate strategies for the management of typhoid fever.

\section{Objectives}

a) General objective:

To examine the clinical profile of patients with typhoid fever and sensitivity patterns of isolated S. typhi.

b) Specific Objectives:

1) Demographic characteristics of the patients with typhoid fever.

2) Clinical profile of the patients with typhoid fever.

3) The antimicrobial susceptibility pattern of the isolated $S$. typhi.

\section{Methodology and Materials}

This was a Cross-sectional observational study. The study was carried out under the department of Pediatrics of Bangabandhu Sheikh Mujib (BSMMU) Medical University, Dhaka Medical College Hospital (DMCH) and Sir Salimullah Medical College Hospital (SSMCH). From these areas, 75, 10 and 15 cases were selected respectively. In the above centers, a lot of pediatric patients with typhoid fever are admitted every year for evaluation and management. Overall, period of study was July 2015 to June 2016 (One year). Ethical clearance was taken from Institutional Review Board (IRB) of BSMMU and the participants were explained that there will be no physical or social risk. The. Children admitted in the Pediatrics wards of the above mentioned hospital during the study period with suspected diagnosis of typhoid fever were the study population. Children with positive blood culture were diagnosed as 'confirm case' and cases with a titer of TO $\geq 1: 160$ or rising titer of Wedel test were diagnosed as 'probable case'. All children with diagnosis of 'confirmed' or 'probable cases' admitted in those hospital during the study period were the sample. During the study period febrile patients admitted in above mentioned hospital with the diagnosis of suspected typhoid fever was screened. Suspicion was made on the basis of clinical features like continued fever, toxic look, diarrhea/constipation, splenomegaly, hepatomegaly, diffuse tenderness and caecal gurgling. Clinically suspected cases undergone laboratory investigation that includes $\mathrm{CBC}$, Widal test and blood culture and other tests as per individual case merits. To determine the sample size the following formula 100 children with the diagnosis of typhoid fever and fulfilling the inclusion and exclusion criteria's during the study period were selected purposively.

Inclusion Criteria

1) Patient diagnosed as typhoid fever and admitted in mentioned hospitals during the study period.

2) Pediatric age group of both sexes

Exclusion Criteria

1) Patient with severe co-morbidities unrelated to typhoid fever.

2) Patient with paratyphoid fever.

3) Care-giver not willing to give written consent

\section{Results}

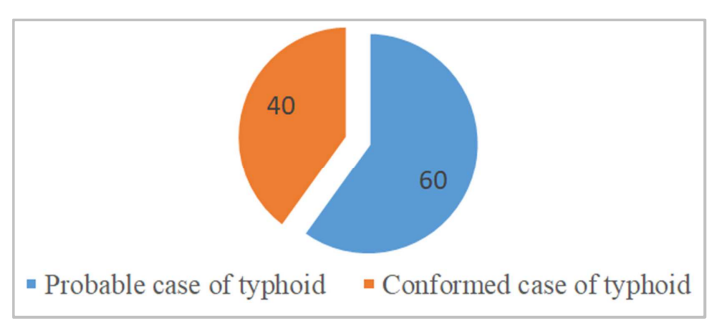

Figure 1. Distribution of typhoid patient according to case definition $(n=100)$.

A total number of 100 children's were taken for the study. (Figure 1) showed, out of 100 patient $60 \%$ were probable case and $40 \%$ were confirmed case of typhoid fever. (Table 1) showed homogenous distribution of cases amongst the three age groups with a male to female. Children from 1-5 years (37\%), from 5-10 years (35\%) and above 10 years (28\%) were studied for this case. (Figure 2) showed, BMI of studied 100 children showed that majority $(65 \%)$ were in the normal range of nutritional status. (Figure 3) showed, predominant sign was coated tongue $(65 \%)$ followed by hepatomegaly $(63 \%)$ and splenomegaly (38\%). Abdominal tenderness was found in $25 \%$ of children. (Table 2) revealed significant number $(20 \%)$ isolates were still MDR while maximum number (90\%) isolates were NARST. (Figure 4) showed, sensitivity pattern of Salmonella typhi. Highest $(100 \%)$ in case of sensitivity was seen with Ceftriaxone followed by Cefixime, Ciprofloxacin, Azithromycin, Chloramphenicol, Cotrimoxazole, Amoxicillin and lowest (10\%) in Nalidixic acid. (Table 2) showed most $(86 \%)$ of the children had abatement of fever $<5$ days while $58.1 \%$ children achieved normal liver span between 3-5 days 
of treatment and mortality was $0 \%$.

Table 1. Age distribution of the studied participants $(n=100)$.

\begin{tabular}{lll}
\hline Age & N & \% \\
\hline 1-5 years & 37 & 37 \\
5-10 year & 35 & 35 \\
$>10$ year & 28 & 28 \\
\hline
\end{tabular}

Table 2. Percentages of MDR \& NARST in isolated S. typhi $(n=40)$.

\begin{tabular}{lll}
\hline Isolates & Number of children & $\%$ \\
\hline MDR & $08(40)$ & 20 \\
NARST & $36(40)$ & 90 \\
\hline
\end{tabular}

MDR- Multi-drug resistant typhoid, NARST- Nalidixic-Acid Resistant Salmonella Typhi

$\%$

$>95$ th centile

85 th $-<95$ th centile

5 th- $<85$ th centile

$<5$ th centile

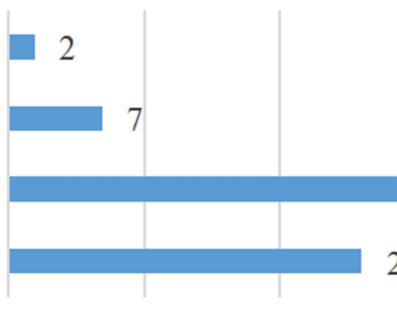

$\begin{array}{llllllll}0 & 10 & 20 & 30 & 40 & 50 & 60 & 70\end{array}$

Figure 2. BMI of the studied children with typhoid fever $(n=100)$.

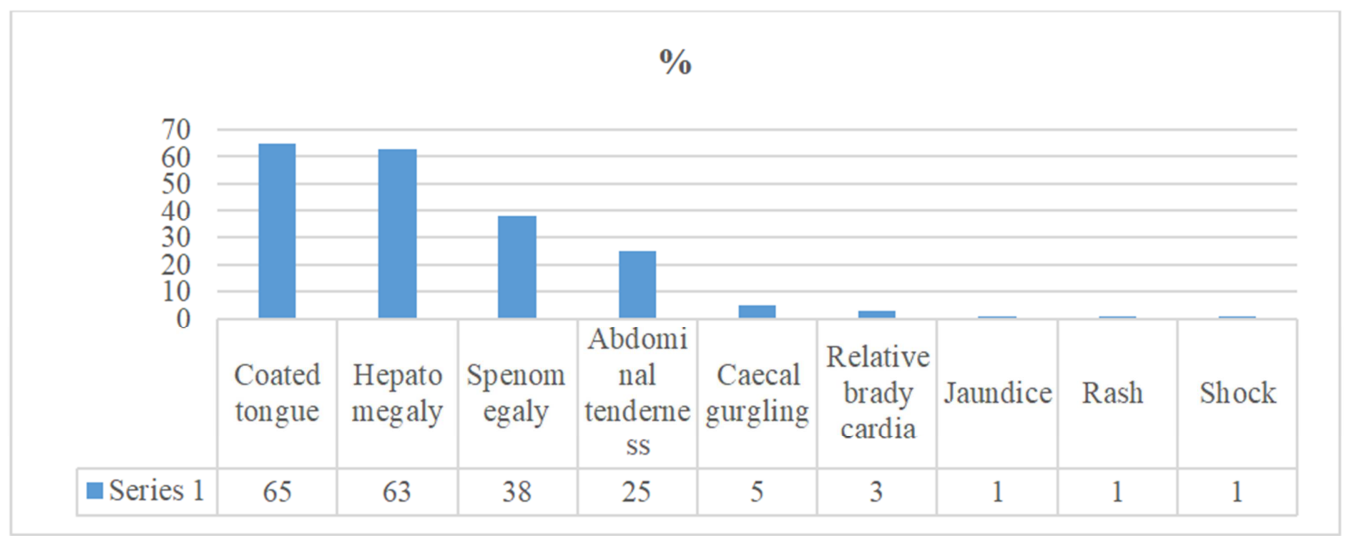

Figure 3. Physical findings of children with typhoid fever $(n=100)$.

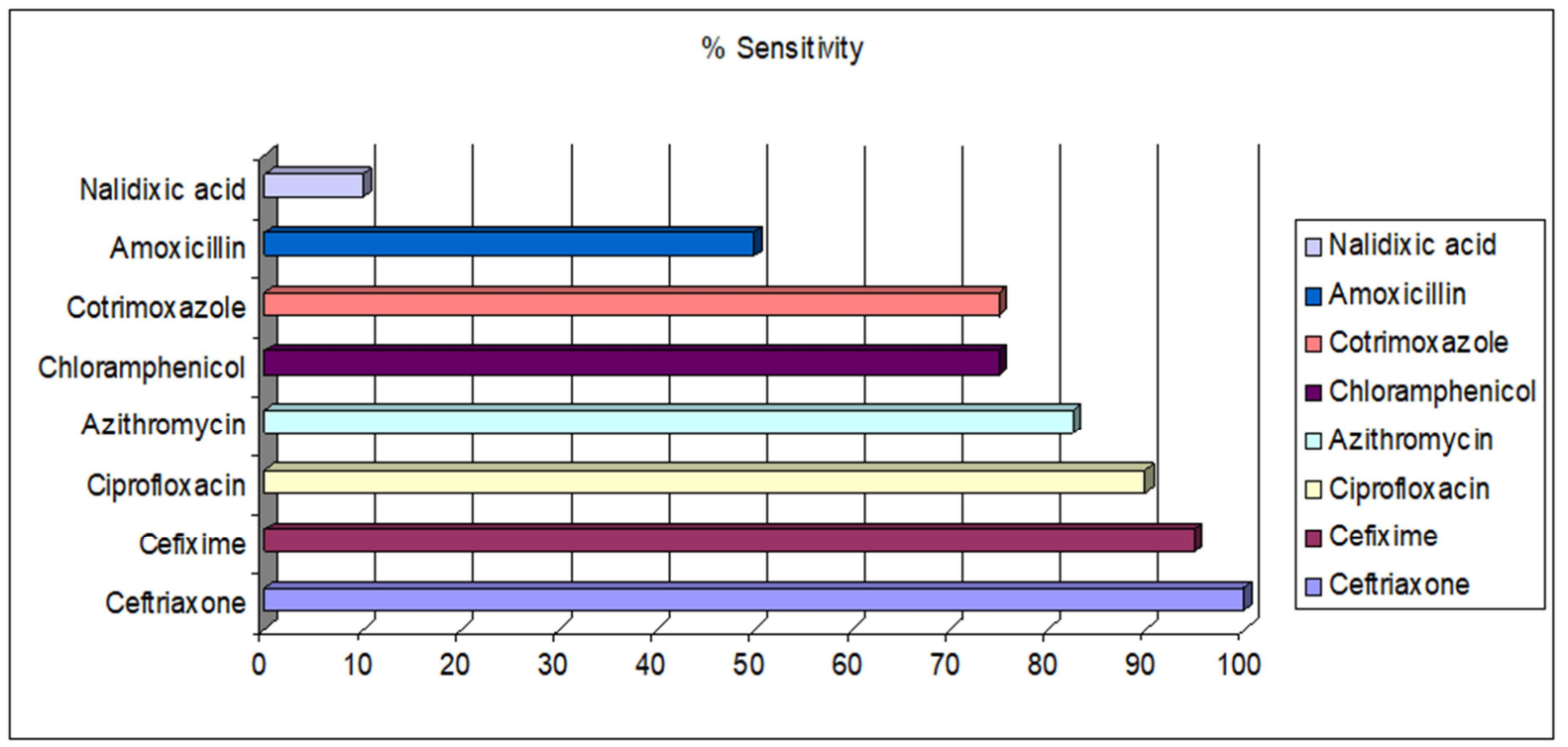

Figure 4. Antimicrobial sensitivity patterns of isolated S. typhi. $(n=40)$. 


\section{Discussion}

Typhoid fever is endemic in our country and affect all ages beyond infancy as found in this study. Similar findings were reported $[2,7]$ This may be due to poor sanitary condition and non-availability of safe water. The present study showed male preponderance 1.5:1. This is consistent with the result. $[8,9]$ However, other report suggests similar prevalence amongst both sexes. [10] Probable explanation might be that in a male dominant society like ours male child has higher access to medical care. It may also be due to the fact that the male children are more independent and has more chance of exposure to street food. Many studies showed higher prevalence amongst poor socioeconomic background. But in the present study the distribution was quite even in all three economic categories. This may suggest that the division was inappropriate or the availability of safe water or exposure to contaminated food was wide and rampant. High percentage of cases in children with normal range of BMI in present study may also support the above. Most $(87 \%)$ of the children had improved sanitation facility in their home but needs to be cent percent. According to BBS 2014 report the distribution of sanitation in Dhaka division were improved sanitation $70 \%$, unimproved sanitation $29 \%$, others $1 \%$. Though the use of improved sanitation in case of typhoid patient were similar to general population in Dhaka but percentage of hand washing seems to be less. In our study sensitivity pattern of salmonella typhi to first line conventional antimicrobials showed sensitivity to Amoxicillin (50\%), Chloramphenicol (75\%), Cotrimoxazole (75\%). Abdullah Brooks et al. (2005). [9] in their study in Bangladesh S. Typhi was isolated from 27 children and revealed sensitivity to Amoxicillin (55.1\%), Chloramphenicol (57.1\%) and Cotrimoxazole (57.1\%) [11] in their study of 68 isolated S. typhi from children, revealed sensitivity to amoxicillin $28.3 \%$, cotrimoxazole $27.4 \%$ and chloraphenicol $22.6 \%$. A study of 175 isolated S. typhi in Bangladesh revealed sensitivity to Amoxicillin (33\%), Chloramphenicol (65.7\%), Cotrimoxazole (64\%) $[7,12]$ et al. (2015) in their study of 56 isolates from children at ICDDRB revealed sensitivity to Amoxicillin (66\%), Chloramphenicol (76.78\%) and Cotrimoxazole $(76.78 \%)$. The reason of reemergence of sensitivity of Chloramphenicol and Cotrimoxazole that these drugs are less frequently being used in children now a days. In present study out of 40 isolates, 08 $(20 \%)$ isolates were MDR strains. Among the isolates from Bangladesh, $82 \%$ and $40 \%$ were resistant to various concentrations of nalidixic acid and ciprofloxacin, respectively. Several resistance mechanisms, including alteration in gyrase A, the presence of QnrS and enhanced efflux pumps, were involved in the reduced susceptibility and resistance to fluroquinolones ${ }^{13}$. The majority of S. typhi MDR isolates from Bangladesh belong to haplotype H58, a prevalent MDR clone that has spread over all of Asia and Africa. In more than $50 \%$ of these MDR isolates from Bangladesh, the genes responsible for resistance reside on the chromosome $[13,7]$ in their study of 56 isolates from children at ICDDRB, revealed $14.3 \%$ were MDR. The present study sensitivity to Azithromycin was $82.5 \%$ while in their study of 56 isolates from children had $100 \%$ sensitivity to Azithromycin.

\section{Limitations of the Study}

Study depict picture of limited number of tertiary care hospitals. It may not be the picture of the community as a whole.

\section{Conclusion and Recommendations}

Clinical profile of typhoid fever in children admitted in tertiary care hospitals in Dhaka matches to what had been seen in the past and recorded in textbooks. Nalidixic acid associated resistance was found to be high. However, Ceftriaxone was $100 \%$ sensitive. Moreover, there was resurgence of sensitivity to first-line drugs. Recommendations: Efforts should be taken to find the sensitivity patterns of typhoid fever before starting treatment. Continuous surveillance of typhoid fever and drug sensitivity pattern may be initiated. Community oriented study may be done to know the disease.

\section{References}

[1] Chand HJ, Rijal KR, Neupane B, Sharma VK, Jha B 2014 , 'Re-emergence of susceptibility to conventional first line drugs in Salmonella isolates from enteric fever patients in Nepal'. J Infect Dev Ctries; vol. 8, pp. 1483-1487.

[2] Siddiqui FJ, Rabbani F, Hasan R, Nizami SQ, Bhutta ZA 2006, Typhoid fever in children: Some epidemiological considerations from Karachi, Pakistan. International J Infectious Diseases; vol. 10, pp. 215-22. (Kothari, 2008 and Cho, 19)

[3] Bhan MK, Bahl R, Bhatnagar S 2005, 'Typhoid and paratyphoid fever'. Lancet; vol. 366, pp. 749-762.

[4] Arora D, Singh R, Kaur M, Ahi RS 2010, 'A changing pattern in antimicrobial susceptibility of Salmonella enterica serotype isolated in north India'. Afr J Microbiol Re; vol. 4, pp. 197203.

[5] Muthu G, Suresh A, Sumathy G, Srivani R 2011, 'Studies on antimicrobial susceptibility pattern of Salmonella isolates from Chennai, India'. Int J Pharma Bio Sci; vol. 2, pp. 435442 .

[6] World Health Organization 2003. 'Background document: the diagnosis, treatment and prevention of typhoid fever'. Geneva: WHO 2003.

[7] 1m F, Sayeed MA, Choudhury FK, Sheikh A, Ahmed D, Goswami D, et al., 2015, Typhoid Fever in Young Children in Bangladesh: Clinical Findings, Antibiotic Susceptibility Pattern and Immune Responses. PLoS Negl Trop Dis; vol. 9, pp. 3619. 
[8] Kumar S, Rizvit M, and Berry N. 2008, Rising prevalence of enteric fever due to multidrug resistant salmonella: an epidemiological study. J. Of medical microbiology; vol. 57, pp. 1247-50.

[9] Abdullah FE, Haider F, Fatima K, Irfan S, Iqbal MS 2012, Enteric Fever in Karachi: Current Antibiotic Susceptibility of Salmonellae Isolates. Journal of the College of Physicians and Surgeons Pakistan; vol. 22, pp. 147-150.

[10] Shah G, Ghimire A, Shrestha S 2014, Clinical profile of culture proven enteric fever in children at university teaching hospital, Nepal. Journal of Patan Academy of Health Sciences; vol. 1, pp. 42-45.
[11] Alam ABMS, Zaman S, Chaiti F, Sheikh N, Kundu GK 2010, A Reappraisal of Clinical Characteristics of Typhoid Fever. Bang J Child Health; vol. 34, pp. 80-85.

[12] Chowdhury MAJ, Shumy F, Anam, AM, Chowdhury, MK 2014, Current status of typhoid fever: a review. Bangladesh Med J; vol. 43, pp. 106-111.

[13] Chiou SC, Lauderdale TL, Phung DC et al, 2014 'Antimicrobial Resistance in Salmonella enteric Serovar Typhi Isolates from Bangladesh, Indonesia, Taiwan, and Vietnam.' Antimicrobial Agents and Chemotherapy; vol. 58, no. 11, pp. 6501-6507. 